\title{
Preditores da empregabilidade individual de profissionais em transição de carreira
}

\author{
Daniela Boucinha ${ }^{1}$, Alexsandro Luiz De Andrade ${ }^{2}$, Diana Aguiar Vieira ${ }^{3}$ \& Manoela \\ Ziebell de Oliveira ${ }^{1}$ \\ 1 Pontifícia Universidade Católica do Rio Grande do Sul \\ 2 Universidade Federal do Espírito Santo \\ ${ }^{3}$ CEOS.PP, ISCAP, Politécnico do Porto
}

\begin{abstract}
Resumo: Os novos paradigmas económicos e tecnológicos imprimem a necessidade dos indivíduos se manterem ativos, adaptáveis às mudanças do mercado de trabalho e capazes de implementar estratégias de promoção de empregabilidade. Este estudo tem como objetivo investigar variáveis individuais que possam ser preditoras da empregabilidade de profissionais em transição. Participaram 181 sujeitos que responderam a um questionário online de avaliação da empregabilidade, adaptabilidade de carreira, decisão de carreira, autorreflexão e insight. Os resultados indicaram que o insight, as dimensões controlo e confiança da adaptabilidade de carreira e a decisão de carreira foram capazes de predizer a empregabilidade, explicando $51 \%$ da variância do modelo. Tais resultados corroboram estudos anteriores, mas também acrescentam a importância que o insight assume no desenvolvimento da empregabilidade individual. Em termos de implicações para a prática, este estudo contribui para munir os profissionais desta área de um maior conhecimento acerca dos preditores da empregabilidade individual nas transições de carreira.
\end{abstract}

Palavras-chave: Adaptabilidade de carreira; decisão de carreira; empregabilidade; insight; preditores; transição de carreira.

Predictors of individual employability among professionals in career transition: The new economic and technological paradigms imply the need for individuals to remain active, adaptable to constant changes in the labor market and capable of implementing employability promotion strategies. This study aimed at investigating predictors of employability among professionals in career transition. Participants were 181 individuals who answered an online questionnaire that assessed employability, career adaptability, career decision, self-reflection and insight. Results indicated that employability was predicted by insight, control, confidence and career decision, that together explained $51 \%$ of the total variance. These results corroborate previous studies but also add the importance that insight assumes in the development of individual employability. In terms of implications for practice, this study contributes to providing professionals in this area with greater knowledge about the predictors of individual employability in career transitions.

Keywords: Career adaptability; career decision; career transition; employability; insight; predictors.

Até à década de 1960 o conceito de carreira associava-se à estabilidade, à progressão linear, às relações de trabalho de longo prazo e à lealdade mútua entre os indivíduos e as organizações (Baruch et al., 2015). No cenário atual, marcado pela volatilidade, incerteza, complexidade e ambiguidade, a carreira é caracterizada pela inconstância, impermanência e aumento da mobilidade (Ambiel, 2014). Assim, para que as organizações sejam competitivas, e para que os profissionais consigam manter-se ativos, sobreviver às constantes mudanças do mercado de trabalho e alcançar uma carreira de sucesso, é necessário investir na empregabilidade individual bem como em políticas organizacionais e públicas de formação e desenvolvimento profissional (Duffy et al., 2016).

O interesse pelo fenómeno da empregabilidade ganhou especial destaque a partir de 1990, quando surgiram diversos estudos sobre o tema, continuando, ainda hoje, a ser um dos tópicos mais recorrentes nas investigações sobre carreira (Baruch et al., 2015). Apesar disso, compreender a relação entre as

\footnotetext{
${ }_{1}^{1}$ Morada para correspondência: Avenida Ipiranga 6681- Prédio 11, Sala 902 - Partenon, Porto Alegre - Rio Grande do Sul, 90619-900, Brasil. E-mail: danielaboucinha.coach@gmail.com

o presente trabalho foi realizado com apoio da Coordenação de Aperfeiçoamento de Pessoal Nível Superior - Brasil (CAPES) - Código de Financiamento 001.
} 
características dos profissionais e as expectativas do mundo do trabalho de forma mais aprofundada constitui-se, ainda, como um desafio (Guilbert et al., 2016).

De maneira geral, a empregabilidade refere-se à capacidade de movimentação e ao conhecimento sobre o mercado de trabalho. Tal capacidade se relaciona com a forma como os profissionais utilizam os seus recursos - conhecimentos, habilidades e atitudes -, o modo como os apresentam aos empregadores (Sok et al., 2013) e como gerem de forma autónoma as questões associadas ao risco e à insegurança, numa economia global (Parker, 2008).

Na perspetiva de Campos e Freitas (2008), pode-se entender a empregabilidade a partir das competências e habilidades necessárias para que o indivíduo conquiste e mantenha um trabalho ou emprego. Tal definição remete para a eficácia nos resultados na procura de emprego ou trabalho, assim como para as características pessoais, crenças e atitudes que poderão afetar a empregabilidade dos indivíduos (Campos \& Freitas, 2008). Com efeito, pessoas com mais empregabilidade tendem a analisar e procurar alternativas laborais compatíveis com as suas identidades de carreira, mostrando-se mais predispostas a realizar mudanças sempre que necessário (Fugate et al., 2004). Neste enquadramento, a capacidade de refletir sobre si próprio e de compreender as suas próprias características contribui de forma inequívoca para a construção de uma identidade de carreira robusta capaz de orientar a exploração do mundo do trabalho (Leal et al., 2018).

Num estudo sobre a empregabilidade percebida, entendida como a perceção de facilidade na obtenção de um novo emprego, Berntson e colaboradores (2006) cruzaram dados relacionados com o capital humano e com as condições do mercado de emprego. Os autores concluíram que tanto os aspetos estruturais do sistema económico-laboral (por exemplo, o mercado de trabalho, o crescimento da economia) quanto os aspetos individuais (ex. autogestão, proatividade) influenciam a empregabilidade percebida.

Embora a empregabilidade seja entendida como a combinação de aspetos educacionais, governamentais, organizacionais e individuais (Guilber et al., 2016), a intervenção nesta área foca-se no indivíduo e visa promover a agência pessoal na construção da carreira (Rothwell et al., 2008).

A literatura indica que as transições de carreira serão cada vez mais frequentes e que a vivência bem-sucedida das transições de carreira é vista como altamente dependente da empregabilidade do indivíduo (Baruch et al., 2015). No entanto, há pouca evidência sobre o modo como a empregabilidade é mantida e desenvolvida, especialmente em contextos de transição. Num estudo com profissionais em transição de carreira, Clarke (2007) concluiu que na mudança de uma carreira estável dentro de uma organização para um mercado de trabalho incerto, os participantes apresentam muita dificuldade em identificar o que podem oferecer a um empregador e evidenciar as suas habilidades e experiência, bem como quanto ao modo de procurar emprego.

Tendo em vista o impacto da empregabilidade para os resultados individuais e organizacionais, bem como a sua relevância para a construção e transições de carreira dos trabalhadores, este estudo tem como objetivo investigar variáveis individuais que possam ser preditoras da empregabilidade de profissionais em transição. No âmbito das variáveis individuais potencialmente preditoras da empregabilidade, no presente estudo serão consideradas a autorreflexão e o insight (DaSilveira et al., 2012; Leal et al., 2018), a adaptabilidade de carreira (Savickas, 2013) assim como a decisão de carreira (Teixeira \& Gomes, 2005).

\section{Autorreflexão, insight e empregabilidade}

Segundo Grant e colaboradores (2002), a autoconsciência é composta por duas dimensões: a autorreflexão e o insight. Estes autores defendem que a autorreflexão diz respeito ao reconhecimento e avaliação dos próprios pensamentos, sentimentos e comportamentos. Por seu turno, o insight consiste na clareza de entendimento sobre os próprios pensamentos, sentimentos e comportamentos (Grant et al., 2002; Leal et al. 2018). 0 insight consiste na habilidade do indivíduo para, a partir da autorreflexão, estabelecer julgamentos, realizar autoavaliações e autorregular-se (Stein \& Grant, 2014). Trata-se, portanto, da tendência do indivíduo para, ao direcionar-se intencionalmente para a mudança, entender com clareza aspetos de si mesmo e das situações com que se depara (Stein \& Grant, 2014). É importante, contudo, destacar que um indivíduo pode despender muito tempo a refletir sobre si (autorreflexão) sem necessariamente alcançar um entendimento esclarecedor sobre a própria experiência (insight) (DaSilveira et al., 2012; Leal et al., 2018).

Mais especificamente, a literatura na área da gestão da carreira indica que os indivíduos que refletem com maior frequência sobre os seus pensamentos têm mais sucesso na clarificação dos seus objetivos e projetos profissionais e maior proatividade na gestão das suas carreiras (Oliveira \& Gomes, 2014). 0 processo de pensar e ter consciência sobre si é, portanto, fundamental na construção de carreira, devendo as intervenções em contextos de orientação profissional e aconselhamento de carreira ter em 
conta a importância do processo de autorreflexão sobre as próprias competências (Borges et al., 2015). Efetivamente, os clientes em processo de aconselhamento de carreira são incentivados a pensar sobre o significado dos acontecimentos das suas vidas, numa perspetiva de análise e reflexão crítica, desenvolvendo o autoconhecimento, aprofundando a autoexploração e facilitando o planeamento de carreira (Cardoso et al., 2016).

Estudos acerca da autoconsciência e, mais precisamente, sobre a capacidade de autorreflexão e insight evidenciam que estas variáveis se associam à obtenção de melhores desempenhos académicos e profissionais (Leal et al., 2018). De destacar que a capacidade para refletir sobre si mesmo é uma característica essencial para que os indivíduos possam gerir as suas carreiras (Zanon \& Teixeira, 2006). Ainda, Pool e Sewel (2007) referem que a tomada de consciência e avaliação das experiências de aprendizagem só é conseguida através da capacidade de autorreflexão e de insight. Assim, a primeira hipótese (H1) deste estudo propõe que: A autorreflexão e o insight possuem um papel preditor da empregabilidade dos profissionais em transição de carreira.

\section{Adaptabilidade de Carreira e Empregabilidade}

A adaptabilidade de carreira é constituída por quatro dimensões: a) preocupação (concern), que diz respeito à avaliação do indivíduo sobre o próprio futuro como trabalhador, o que promove atitudes de planeamento; b) controlo (control), que se refere à responsabilidade de construir a própria carreira, o que implica assumir uma maior responsabilidade nas escolhas de carreira e no futuro profissional; c) curiosidade (curiosity), relacionada com a exploração de oportunidades e atividades laborais nas quais o indivíduo gostaria de se envolver, requerendo o conhecimento acerca de si mesmo e do mundo de trabalho; e, d) confiança (confidence), relacionada com a crença do indivíduo na sua eficácia para atingir os seus objetivos, mesmo quando confrontado com obstáculos (Savickas, 2013).

Assim, a adaptabilidade de carreira é um construto importante a considerar durante as transições de carreira uma vez que as dimensões da adaptabilidade de carreira promovem a construção dos recursos necessários para que os indivíduos possam assumir a gestão de suas próprias carreiras, e para que os orientadores de carreira possam entender os padrões das estratégias utilizadas pelos indivíduos para lidar com as barreiras profissionais (Audibert \& Teixeira, 2015). Adicionalmente, a investigação nesta área sugere que os indivíduos que apresentam maior adaptabilidade de carreira tendem a aproveitar mais os recursos do seu contexto (Rossier, Ginevra, Bollmann, \& Nota, 2017), o que acaba por contribuir para o aumento da sua empregabilidade (Fraga, 2012). Efetivamente, Fugate et al. (2004) defendem que a empregabilidade pode ser entendida como uma forma específica de adaptabilidade ativa que permite aos trabalhadores identificarem e concretizarem oportunidades de carreira o que, por sua vez, facilita a movimentação entre postos de trabalho, dentro e fora das organizações.

Considerando que indivíduos com maior adaptabilidade de carreira tendem a manifestar maior empregabilidade (Fraga, 2012; Rossier et al., 2017) e que as quatro dimensões operacionais deste construto se relacionam com a agência individual da empregabilidade (Gamboa et al., 2014), propõe-se a seguinte hipótese: (H2) A adaptabilidade de carreira é preditora da empregabilidade dos profissionais em transição de carreira.

\section{Decisão de carreira e Empregabilidade}

A decisão de carreira é entendida como a clareza dos objetivos, interesses e talentos profissionais, bem como a capacidade de identificar interesses profissionais, estabelecer objetivos profissionais e implementar estratégias de ação face aos projetos profissionais, não se limitando à escolha de uma área profissional específica (Teixeira \& Gomes, 2005). Como construto, a decisão de carreira é um indicador da capacidade de antecipação e planeamento das ações necessárias para conseguir atingir objetivos de carreira (Teixeira et al., 2019).

Conforme Bardagi e Hutz (2010), ter metas e planos definidos associa-se a uma maior satisfação com a vida, um maior comportamento exploratório bem como um maior comprometimento com a carreira. Teixeira (2002) reforça que os indivíduos que encaram o futuro profissional de forma mais otimista sentem-se mais motivados para enfrentar eventuais dificuldades e para traçar metas profissionais num mercado de trabalho marcado pela imprevisibilidade.

Considerando que decisão de carreira é uma atitude individual de afirmação em relação ao projeto profissional, representada pela capacidade de definir metas e planos a partir da clareza e certeza sobre um objetivo profissional (Teixeira et al., 2019) e que o envolvimento contínuo em processos de avaliação e de tomada de decisão contribui para o aumento da empregabilidade (Bridgstock, 2009), formulou-se a seguinte hipótese: (H3) A decisão de carreira é preditora da empregabilidade dos profissionais em transição de carreira. 


\section{MÉTODO}

\section{Participantes}

0 presente estudo contou com uma amostra de 181 participantes voluntários que procuraram o serviço de consultoria de carreira de uma universidade localizada na região Sul do Brasil, sendo 102 (56\%) mulheres e 79 (44\%) homens. A média de idade dos participantes foi de 29 anos ( $D P=7$ anos), 71\% solteiros e $81 \%$ moravam com os pais. Em relação à escolaridade, 81 participantes concluíram o ensino superior (45\%), 57 frequentavam o ensino superior (32\%) e 39 concluíram pós-graduações (23\%).

\section{Instrumentos}

Para a recolha dos dados foi construído um formulário online subdividido em duas partes: a) uma secção para a caracterização do perfil sociodemográfico dos participantes (idade, sexo, rendimentos, estado civil, número de filhos, formação académica e situação face ao trabalho); e b) uma segunda parte com quatro instrumentos psicométricos apresentados seguidamente.

Escala de Autorreflexão e Insight. Criada por Grant et al. (2002) e adaptada e validada para utilização no Brasil por DaSilveira e colaboradores (2012), esta escala bidimensional é composta por 20 itens com um formato de resposta Likert de cinco pontos. A dimensão "autorreflexão" (12 itens; $\alpha=0.90$ ) refere-se à capacidade de inspecionar e avaliar pensamentos, sentimentos e comportamentos (por exemplo: "Eu frequentemente penso sobre como me sinto sobre as coisas"). A dimensão "insight" (8 itens; $\alpha=0.82$ ) refere-se ao entendimento interno que se tem sobre os seus pensamentos, sentimentos e comportamentos (por exemplo: "Eu normalmente sei por que me sinto da forma como me sinto").

Escala de Decisão de Carreira. Este instrumento unidimensional foi desenvolvido por Teixeira (2002) e é constituído por dez itens respondidos numa escala Likert de cinco pontos $(\alpha=0.90)$. A escala avalia a clareza do indivíduo quanto à definição dos seus objetivos e metas profissionais (por exemplo: "Eu tenho clareza sobre quais são os meus objetivos profissionais").

Escala de Adaptabilidade de Carreira. A versão original desta escala (Career Adapt-Abilities Scale) foi desenvolvida por Savickas e Porfeli (2012). A adaptação para o contexto do Brasil foi realizada por Audibert e Teixeira (2015). A Escala de Adaptabilidade de Carreira é composta por 24 itens que são respondidos numa escala Likert de cinco pontos. Os itens abrangem questões relacionadas com as quatro dimensões da adaptabilidade de carreira: a) "preocupação" (6 itens; $\alpha=0.88$ ) que avalia a reflexão do indivíduo sobre o seu futuro profissional (por exemplo: "Pensar sobre como será o meu futuro"); b) "controlo" (6 itens; $\alpha=0.83$ ) que avalia a capacidade do indivíduo para construir a sua carreira (por exemplo: "Assumir responsabilidade pelos meus atos"); c) "curiosidade" ( 6 itens; $\alpha=0.88$ ) que avalia a capacidade do indivíduo para explorar e procurar novos conhecimentos e aprendizagens (por exemplo: "Explorar as opções antes de fazer uma escolha"); e, d) "confiança" (6 itens; $\alpha=0.89$ ) que avalia a crença do indivíduo em alcançar os seus objetivos profissionais (por exemplo: "Superar obstáculos").

Escala de Empregabilidade. Este instrumento foi desenvolvido por Campos e Freitas (2008) e é composto por 57 itens respondidos numa escala Likert de quatro pontos. A escala apresenta quatro dimensões: a) "eficácia de busca" (22 itens; $\alpha=0.88$ ) - avalia sentimentos positivos face a capacidade de realizar as atividades necessárias para conseguir um emprego (por exemplo: "Sinto-me preparado para participar de entrevistas e avaliações de trabalho"); b) "dificuldade de busca" (10 itens; $\alpha=0.71$ ) - traduz a perceção de falta de empregabilidade relacionada com um ânimo negativo (por exemplo: "No geral, eu não espero ser muito bom na busca de um emprego/trabalho"); c) "otimismo" (16 itens; $\alpha=0.84$ ) - reflete sentimentos de autoconfiança (por exemplo: "Eu tenho uma atitude positiva quanto a mim mesmo"); e, d) "responsabilidade e decisão" ( 9 itens; $\alpha=0.72$ ) - refere-se a aspetos ligados à persistência, decisão, confiança e ao autoconhecimento (por exemplo: "Escolhi uma carreira que combina com minhas habilidades").

\section{Procedimentos de Recolha de dados}

A recolha de dados foi realizada através de um questionário online cujo acesso foi disponibilizado no âmbito de um serviço de orientação e planeamento de carreira de uma instituição de ensino superior da região Sul do Brasil. Este serviço tem como público-alvo alunos e diplomados de diferentes cursos de graduação. 0 cálculo amostral considerou o número total de indivíduos atendidos no serviço no ano anterior, e adotou como parâmetros 5\% de estimativa de erro amostral e um nível de confiança de 95\%. 
Durante a primeira entrevista aos clientes do serviço, identificou-se o motivo da procura por este tipo de intervenção. As necessidades identificadas foram classificadas como "apoio à recolocação" ou "planeamento de carreira" e, em casos específicos, "planeamento e recolocação". Os clientes com mais de 18 anos que estavam à procura de recolocação foram convidados a participar da pesquisa. 0 questionário online incluiu o termo de consentimento livre e esclarecido, informando que o fornecimento dos dados para o estudo seria voluntário e que não acarretaria mudanças na forma como seriam atendidos no serviço. Depois de recebermos as respostas, os dados foram armazenados numa base de dados sem a identificação dos participantes.

\section{Procedimento de Análise de dados}

Os dados foram analisados com o auxílio do software estatístico R (R Development Core Team, 2010). Inicialmente, foram realizadas análises descritivas e de frequências de todos os itens e variáveis. Seguidamente foram analisados e excluídos os dados discrepantes, bem como calculadas as médias das dimensões nas escalas psicológicas utilizadas. Os procedimentos de correlação e de regressão hierárquica foram realizados com o método Enter para testar as hipóteses do presente estudo e identificar as variáveis preditoras da empregabilidade individual.

\section{RESULTADOS}

A Tabela 1 apresenta as estatísticas descritivas (média e desvio-padrão) e a matriz de correlação entre a pontuação total da empregabilidade (empregabilidade geral) e as suas dimensões, autorreflexão e insight, decisão de carreira, a pontuação total da adaptabilidade de carreira (adaptabilidade de carreira geral) e as suas respetivas dimensões.

Tabela 1. Matriz de Correlação das Variáveis de Decisão de Carreira, Empregabilidade Geral e Suas Dimensões, Adaptabilidade Geral e Suas Dimensões, Autorreflexão e Insight

\begin{tabular}{|c|c|c|c|c|c|c|c|c|c|c|c|c|c|}
\hline & 1 & 2 & 3 & 4 & 5 & 6 & 7 & 8 & 9 & 10 & 11 & 12 & 13 \\
\hline 1. Decisão de carreira & - & & & & & & & & & & & & \\
\hline 2. E - Eficácia de busca & $.46^{* *}$ & - & & & & & & & & & & & \\
\hline 3. E - Dificuldade de busca & $-.31^{* *}$ & $.41^{* *}$ & - & & & & & & & & & & \\
\hline 4. E- Otimismo & $.15^{*}$ & $.35^{* *}$ & $-.39 * *$ & - & & & & & & & & & \\
\hline $\begin{array}{l}\text { 5. E - Responsabilidade e } \\
\text { decisão }\end{array}$ & $.47^{* *}$ & $.44^{* *}$ & $-.35^{* *}$ & $.35^{* *}$ & - & & & & & & & & \\
\hline 6. AC - Preocupação & $.44^{* *}$ & $.37^{* *}$ & $.29^{* *}$ & $.17^{*}$ & $.36^{* *}$ & - & & & & & & & \\
\hline 7. AC - Controlo & $.19^{* *}$ & $.35^{* *}$ & $-.40^{* *}$ & $.45^{* *}$ & $.43^{* *}$ & $.40^{* *}$ & - & & & & & & \\
\hline 8. AC - Curiosidade & $.25^{* *}$ & $.30^{* *}$ & $-.30^{* *}$ & $.35^{* *}$ & $.28^{* *}$ & $.57^{* *}$ & $.53^{* *}$ & - & & & & & \\
\hline 9. AC - Confiança & .08 & $.22^{* *}$ & $-.34^{* *}$ & $.38^{* *}$ & $.43^{* *}$ & $.37^{* *}$ & $.54^{* *}$ & $.57^{* *}$ & - & & & & \\
\hline $\begin{array}{l}\text { 10. Adaptabilidade de Carreira } \\
\text { G. }\end{array}$ & $.32^{* *}$ & $.40^{* *}$ & $-.42^{* *}$ & $.42^{* *}$ & $.47^{* *}$ & $.76^{* *}$ & $.78^{* *}$ & $.85^{* *}$ & $.76^{* *}$ & - & & & \\
\hline 11. Empregabilidade geral & $.46^{* *}$ & $.82^{* *}$ & $-.65^{* *}$ & $.74^{* *}$ & $.66^{* *}$ & $.39 * *$ & $.54^{* *}$ & $.42^{* *}$ & $.44^{* *}$ & $.57^{* *}$ & - & & \\
\hline 12. Autorreflexão & -.10 & -.36 & -.30 & .12 & $.19^{*}$ & $.16^{*}$ & .08 & .14 & $.23^{* *}$ & $.19^{*}$ & .76 & - & \\
\hline 13. Insight & $.34^{* *}$ & $.33^{* *}$ & $-.47^{* *}$ & $.40^{* *}$ & $.44^{* *}$ & $.31^{* *}$ & $.45^{* *}$ & $.35^{* *}$ & $.27^{* *}$ & $.44^{* *}$ & $.54 * *$ & .14 & - \\
\hline $\mathbf{M}$ & 2.89 & 2.75 & 2.78 & 3.15 & 3.24 & 3.10 & 3.80 & 3.45 & 4.06 & 3.60 & 2.93 & 4.18 & 3.35 \\
\hline DP & 0.88 & 0.36 & 0.46 & 0.39 & 0.44 & 0.80 & 0.75 & 0.80 & 0.64 & 0.59 & 0.29 & 0.63 & 0.69 \\
\hline
\end{tabular}

Os resultados das análises de correlação indicam que a empregabilidade apresenta correlações positivas e significativas com a decisão de carreira (moderada; $r=0.46$ ), com a adaptabilidade de carreira (moderada; $r=0.57$ ) e suas dimensões, preocupação (moderada; $r=0.39$ ), controlo (moderada; $r=0.54$ ), curiosidade (moderada; $r=0.42$ ), confiança (moderada; $r=0.44$ ), e com o insight (moderada; $r=0.54$ ). Este conjunto de resultados indica que a decisão de carreira, a adaptabilidade de carreira e respetivas dimensões e o insight apresentam uma relação positiva com a empregabilidade, sugerindo que, potencialmente, estas variáveis poderão assumir um papel preditor face à empregabilidade.

\section{Dimensões Preditoras da Empregabilidade}

A Tabela 2 apresenta os resultados das análises de regressão hierárquica realizadas. Foram estabelecidos cinco modelos, os quais consideraram como variável dependente as dimensões da empregabilidade (eficácia de busca; dificuldade de busca; otimismo; responsabilidade e decisão) e a escala geral da 
empregabilidade. Como variáveis independentes foram consideradas as dimensões autorreflexão e insight (bloco 1), a adaptabilidade de carreira (bloco 2) e a decisão de carreira (bloco 3).

Tabela 2. Modelos de Empregabilidade

\begin{tabular}{|c|c|c|c|c|c|c|c|c|c|c|c|c|c|c|c|}
\hline \multirow[b]{2}{*}{ Bloco 1} & \multicolumn{3}{|c|}{ Eficácia de Busca } & \multicolumn{3}{|c|}{$\begin{array}{l}\text { Dificuldade de } \\
\text { busca }\end{array}$} & \multicolumn{3}{|c|}{ Otimismo } & \multicolumn{3}{|c|}{$\begin{array}{l}\text { Responsabilidade- } \\
\text { Decisão }\end{array}$} & \multicolumn{3}{|c|}{$\begin{array}{c}\text { Empregabilidade } \\
\text { Geral }\end{array}$} \\
\hline & $\mathbf{R}^{2}$ & B & Sig. & $\mathbf{R}^{2}$ & B & Sig. & $\mathbf{R}^{2}$ & B & Sig. & $\mathbf{R}^{2}$ & B & Sig. & $\mathbf{R}^{2}$ & B & Sig. \\
\hline Autorreflexão & $11 \%$ & -.09 & .23 & $21 \%$ & -.04 & .56 & $15 \%$ & .06 & .36 & $20 \%$ & .13 & .05 & $29 \%$ & -.00 & .97 \\
\hline Insight & & .34 & .00 & & .47 & .00 & & .39 & .00 & & .42 & .00 & & .54 & .00 \\
\hline Bloco 2 & $\mathbf{R}^{2}$ & B & Sig. & $\mathbf{R}^{2}$ & B & Sig. & $\mathbf{R}^{2}$ & B & Sig. & $\mathbf{R}^{2}$ & B & Sig. & $\mathbf{R}^{2}$ & B & Sig. \\
\hline Autorreflexão & $20 \%$ & -.12 & .08 & $27 \%$ & -.08 & .22 & $26 \%$ & .03 & .61 & $32 \%$ & .07 & .27 & $44 \%$ & -.06 & .34 \\
\hline Insight & & .19 & .01 & & .35 & .00 & & .24 & .00 & & .29 & .00 & & .35 & .00 \\
\hline Preocupação & & .26 & .00 & & .09 & .27 & & -.13 & .10 & & .19 & .01 & & .12 & .08 \\
\hline Controlo & & .16 & .07 & & .14 & .11 & & .25 & .00 & & .15 & .06 & & .24 & .00 \\
\hline Curiosidade & & .01 & .92 & & -.03 & .73 & & .11 & .22 & & -.18 & .04 & & .01 & .91 \\
\hline Confiança & & .01 & .91 & & .17 & .04 & & .15 & .08 & & .28 & .00 & & .18 & .02 \\
\hline Bloco 3 & $\mathbf{R}^{2}$ & B & Sig. & $\mathbf{R}^{2}$ & B & Sig. & $\mathbf{R}^{2}$ & B & Sig. & $\mathbf{R}^{2}$ & B & Sig. & $\mathbf{R}^{2}$ & B & Sig. \\
\hline Autorreflexão & $28 \%$ & -.06 & .37 & $28 \%$ & -.05 & .44 & $26 \%$ & .04 & .51 & $44 \%$ & .15 & .01 & $51 \%$ & .00 & .99 \\
\hline Insight & & .10 & .18 & & .31 & .00 & & .23 & .00 & & .18 & .01 & & .26 & .00 \\
\hline Preocupação & & .12 & .15 & & .02 & .82 & & -.16 & .07 & & .02 & .78 & & -.00 & .96 \\
\hline Controlo & & .18 & .04 & & .15 & .08 & & .25 & .00 & & .17 & .02 & & .26 & .00 \\
\hline Curiosidade & & .00 & .98 & & -.04 & .70 & & .11 & .23 & & -.19 & .02 & & .00 & .98 \\
\hline Confiança & & .04 & .64 & & .19 & .03 & & .16 & .07 & & .32 & .00 & & .20 & .00 \\
\hline $\begin{array}{l}\text { Decisão de } \\
\text { carreira }\end{array}$ & & .33 & .00 & & .16 & .03 & & .06 & .44 & & .40 & .00 & & .26 & .00 \\
\hline
\end{tabular}

A empregabilidade geral foi predita por insight (bloco 1), que explicou 29\% da sua variância, corroborando parcialmente a hipótese 1 . Com o acréscimo das dimensões da adaptabilidade de carreira, controlo e confiança somaram-se ao conjunto das variáveis com poder preditivo, explicando 44\% da variância (bloco 2), dando suporte parcial à hipótese 2. Com o acréscimo da decisão de carreira (bloco 3), o modelo final passou a explicar $51 \%$ da variância da empregabilidade geral e foi predito por insight, controlo, confiança e decisão de carreira, corroborando assim a hipótese 3.

Para uma análise mais detalhada, passamos a apresentar os resultados das regressões hierárquicas considerando cada uma das dimensões da empregabilidade.

A variância da dimensão da empregabilidade "eficácia de busca" foi predita em 11\% pela dimensão insight (bloco 1). Com o acréscimo das dimensões da adaptabilidade de carreira (bloco 2), a dimensão preocupação juntou-se ao insight na predição da "eficácia de busca", somando 20\% de variância explicada. Com o acréscimo da variável decisão de carreira (bloco 3), o modelo final foi capaz de explicar $28 \%$ da variância, sendo predito por: controlo e decisão de carreira. Este resultado sugere que o insight, a preocupação e o controlo são importantes preditores da "eficácia de busca". Contudo, na presença da variável decisão de carreira, apenas a dimensão controlo da adaptabilidade de carreira se mantém relevante.

A dimensão da empregabilidade designada de "dificuldade de busca" foi predita em $21 \%$ por insight de forma negativa (bloco 1). Com o acréscimo das dimensões da adaptabilidade de carreira (bloco 2), a confiança juntou-se ao insight como preditor negativo da "dificuldade de busca", somando $26 \%$ de variância explicada. Com o acréscimo da variável decisão de carreira, o modelo final explicou $28 \%$ da variância da "dificuldade de busca" e foi composto por insight, confiança e decisão de carreira, que foram preditores negativos da "dificuldade de busca".

A dimensão "otimismo" foi predita por insight (bloco 1), que explicou 11\% de sua variância. Com o acréscimo das quatro dimensões da adaptabilidade de carreira (bloco 2), a dimensão controlo juntou-se ao insight na predição do "otimismo", somando $26 \%$ da variância explicada. Com o acréscimo da variável decisão de carreira (bloco 3), não houve alteração no modelo ou na sua capacidade explicativa.

A dimensão "responsabilidade e decisão" foi predita em 20\% por autorreflexão e insight (bloco 1). Com o acréscimo das dimensões da adaptabilidade de carreira (bloco 2), a autorreflexão deixou de ser preditiva e as dimensões insight, preocupação, curiosidade (negativamente) e confiança predisseram 
32\% da "responsabilidade e decisão". Com o acréscimo da variável decisão de carreira (bloco 3), o modelo final explica $44 \%$ da variância da "responsabilidade e decisão" e é composto por autorreflexão, insight, controlo, curiosidade (negativamente), confiança e decisão de carreira.

\section{DISCUSSÃO}

O objetivo deste estudo foi identificar as variáveis preditoras da empregabilidade em profissionais que procuravam uma recolocação no mercado de trabalho, ou seja, indivíduos em transição de carreira.

No que diz respeito ao estudo das relações entre as variáveis consideradas neste estudo, os resultados indicaram que a decisão de carreira apresenta uma relação positiva com três dimensões da adaptabilidade de carreira, nomeadamente preocupação, controlo e curiosidade. Tal resultado indica que quanto mais um indivíduo pensa sobre o seu futuro, explora as suas opções e assume a responsabilidade pela sua carreira, mais tende a sentir-se capaz de tomar decisões. Este resultado vai ao encontro e complementa a literatura que aponta para uma forte relação positiva entre a adaptabilidade de carreira e a tomada de decisão (Creed et al., 2009; Nota et al., 2012; Hirschi et al., 2015; Rossier, 2015). Resultado semelhante foi identificado num estudo com adolescentes italianos no qual se observou o efeito indireto da adaptabilidade de carreira na decisão de carreira através de dois mediadores, atitude positiva face ao futuro e orientação para o futuro (Ginevra et al., 2016). Tendo em vista que a decisão de carreira reflete uma orientação para o futuro, compreender a forma como esta se relaciona com a adaptabilidade de carreira traz benefícios também para a intervenção nesta área. Com efeito, durante os processos de aconselhamento de carreira, os orientadores visam auxiliar os seus clientes na procura de uma visão de futuro satisfatória e de um planeamento de carreira com metas e ações coerentes com as escolhas profissionais do indivíduo.

Os resultados do presente estudo indicaram também que a empregabilidade geral e as suas dimensões apresentam relações positivas com a adaptabilidade de carreira. Em consonância, estudos anteriores já haviam sugerido que os indivíduos que manifestam maior adaptabilidade de carreira tendem a evidenciar maior empregabilidade (Fraga, 2012; Guilbert et al., 2016; Rossier et al., 2017). Este resultado do presente estudo permite considerar a empregabilidade como uma forma de adaptabilidade específica do contexto de trabalho que capacita os indivíduos para identificarem e realizarem ações facilitadoras da movimentação entre trabalhos, quer seja numa organização, quer numa carreira autónoma. Isto é, quanto mais adaptáveis, mais facilmente os indivíduos conseguem adquirir ou manter os seus empregos. Cabe destacar que as estratégias e técnicas para o desenvolvimento da adaptabilidade de carreira, utilizadas por consultores de carreira junto dos seus clientes, tenderão a contribuir para desenvolver a sua empregabilidade.

Sobre as análises preditivas, foi possível identificar importantes preditores da empregabilidade geral, bem como das suas dimensões específicas. Observou-se que a dimensão eficácia de busca foi predita pela decisão de carreira e pela adaptabilidade de carreira, na sua dimensão intitulada de controlo. Os indivíduos que assumem maior responsabilidade nas escolhas e na definição dos seus objetivos face ao seu futuro profissional tendem a traçar estratégias de ação coerentes com tais objetivos e, com isso, conseguem maior êxito na tarefa de busca (Buscacio \& Soares, 2017). Este resultado traz também implicações para a prática de consultoria em carreira. Efetivamente, quanto mais consolidada a escolha e quanto mais o profissional assume para si a responsabilidade pela gestão da sua carreira, mais facilmente encontrará estratégias efetivas para procurar oportunidades (Clarke, 2007). Dessa forma exibirá maior motivação para identificar fontes de oportunidades, preparar o currículo e procurar colocações profissionais mais alinhadas com o seu perfil profissional. Esta ideia é também corroborada pelo estudo de Buscacio e Soares (2017), o qual evidenciou que os estudantes universitários que demonstravam maior clareza quanto ao seu futuro profissional desejado, procuravam e obtinham informações sobre o mercado com maior facilidade.

Outra implicação para a prática resultante do presente estudo diz respeito à eficácia na procura de emprego, bem como à competência na realização de entrevistas e outros contatos de âmbito profissional, os quais requerem clareza sobre a posição desejada. Frequentemente os indivíduos que procuram serviços de apoio à carreira com o objetivo de se inserir no mercado de trabalho, não têm clareza sobre a área na qual gostariam de atuar profissionalmente. Nestes casos, cabe aos consultores de carreira trabalharem, acima de tudo, na definição e clareza das escolhas profissionais, o que se configura como um processo mais reflexivo e de planeamento do que o mero desenvolvimento de técnicas de procura de emprego, o qual, muitas vezes, é o motivo declarado dos clientes que recorrem ao serviço de apoio à carreira. No entanto, como evidencia a literatura, o processo reflexivo favorece as tomadas de decisão de carreira alinhadas com as expectativas e os valores dos profissionais (Clarke, 2007; Oliveira \& Gomes, 2014). 
A dificuldade de busca teve como preditores negativos o insight, a confiança e a decisão de carreira. Ou seja, quanto maiores as pontuações nessas variáveis, menor dificuldade os profissionais terão na busca de oportunidades profissionais. Este resultado vai ao encontro do estudo realizado por Kanfer et al. (2001), no qual o sucesso na busca por trabalho relacionou-se mais com variáveis psicológicas concernentes à afetividade positiva (por exemplo, extroversão e responsabilidade, autoestima e autoeficácia) do que com a afetividade negativa (por exemplo, neuroticismo). Tal como se verificou em relação à eficácia da busca, observou-se que os indivíduos que assumem maior responsabilidade pelas suas trajetórias de carreira e que se comprometem com as suas escolhas tendem a evidenciar menor dificuldade para enfrentar os desafios colocados por um mercado de trabalho turbulento e imprevisível (Clarke, 2007; Oliveira \& Gomes, 2014).

Por sua vez, o otimismo, que se relaciona com a noção de autoconfiança, extroversão e autoestima positiva (Campos \& Freitas, 2008), foi predito por insight e pela adaptabilidade de carreira na sua dimensão de controlo. Pesquisas anteriores já apontavam que as variáveis psicológicas relativas aos afetos positivos influenciam a busca de trabalho (Kanfer et al., 2001). Nesta direção, o otimismo funciona como um componente transformador da empregabilidade dos indivíduos na medida em que envolve a aceitação de si e uma perceção positiva face à transição. Adicionalmente, o otimismo operara como um facilitador do planeamento e da definição de objetivos profissionais.

Já o insight proporciona a tomada de consciência dos pensamentos e sentimentos que os indivíduos têm em relação aos seus comportamentos, escolhas, estratégias e planos profissionais. À medida que os indivíduos assumem maior responsabilidade pela sua empregabilidade, tendem a desenvolver de forma mais intencional e frequente o networking, gerando novos contatos e relações profissionais. A este respeito, a literatura sugere que a mobilização proactiva e intencional do networking pode ajudar a contrabalançar a influência, porventura negativa, de outros fatores contextuais sobre a carreira, tais como o estado da economia e do mercado de trabalho (Rizzatti et al., 2018).

Quanto à dimensão de responsabilidade e decisão, verificou-se neste estudo que a mesma foi predita por autorreflexão, insight, controlo, curiosidade (negativamente), confiança e decisão de carreira. Esta dimensão da empregabilidade reflete a persistência, decisão e escolha da atividade profissional, mas também o autoconhecimento, característica que ajuda na escolha de carreira (Campos \& Freitas, 2008). Pelo facto de o autoconhecimento estar muito associado à responsabilidade e decisão, é interessante observar que esta dimensão da empregabilidade foi a que se relacionou de forma positiva com o maior número de variáveis preditoras consideradas no presente estudo. Na prática da consultoria em carreira, é possível observar que os indivíduos que se envolvem ativamente num processo reflexivo e de autoconhecimento, colocando-se como protagonistas das suas trajetórias comprometem-se mais com as suas escolhas e demonstram maior persistência na busca dos seus objetivos (Oliveira \& Gomes, 2014). É frequente os clientes procurarem serviços de orientação e aconselhamento de carreira com o desejo de definir planos e metas, e surpreenderem-se com a necessidade prévia das tarefas relacionadas com o autoconhecimento. Porém, ao "olhar para si" e se darem conta que o seu funcionamento, sentimentos e motivações influenciam as suas escolhas, os indivíduos conseguem mais facilmente identificar as competências que podem ser úteis no contexto de trabalho. Consequentemente, tendem a sentir-se mais confiantes e a assumir o controlo da sua carreira sob a forma de um planeamento alinhado com projetos de vida mais claros e definidos.

No que diz respeito à empregabilidade geral, os preditores encontrados foram o insight, controlo, confiança e decisão de carreira. Este resultado evidencia a potencial contribuição do aconselhamento de carreira para o desenvolvimento da empregabilidade geral. Através de um processo de aconselhamento de carreira é possível promover a reflexão e a tomada de consciência sobre as trajetórias de vida dos profissionais, a fim de ajudá-los a compreender de que forma constroem as suas próprias vidas, incluindo os seus percursos académicos e laborais. Adicionalmente, segundo Parker (2008), o aconselhamento de carreira auxilia o indivíduo a refletir acerca dos seus recursos e pontos fortes, o que contribui para a construção de uma carreira que expresse o seu autoconceito e a sua identidade profissional. Por outro lado, alguns indivíduos poderão despender muito tempo a refletir sobre si, mas não atingir qualquer insight (Stein \& Grant, 2014). Este tipo de situações reforça a importância de que o apoio às transições de carreira seja desempenhado por profissionais da área de orientação profissional e de carreira. Tais profissionais são capacitados para auxiliar os seus clientes, através da narrativa e de um processo de coconstrução, a vivenciarem uma jornada que envolve o autoconhecimento, a exploração do mundo do trabalho e de possibilidades profissionais até estabelecer um plano de ação coerente com os insights entretanto alcançados. Além disso, os profissionais da área de orientação profissional e de carreira podem incentivar os indivíduos a desenvolverem a capacidade de assumir o controlo das suas próprias carreiras (Clarke, 2007). 
Tal como verificado no estudo de Gamboa e colaboradores (2014), entre as dimensões de adaptabilidade de carreira, o controlo e a confiança foram aquelas que apresentaram relações positivas e mais significativas com a empregabilidade. De acordo com Kanfer e colaboradores (2001), a responsabilidade associada ao controlo e aos resultados tem demonstrado relações positivas com comportamentos de procura de emprego. Já Campos e Freitas (2008) indicam que sentimentos positivos tais como a confiança, extroversão e autoestima se constituem como um fator diferenciador na conquista de um posto de trabalho. Este conjunto de resultados evidencia a importância de os consultores de carreira trabalharem na promoção da confiança dos seus clientes, incrementando a crença na própria competência (autoeficácia) para atingir os seus objetivos, mesmo diante de obstáculos. Neste enquadramento, os consultores de carreira poderão recorrer às fontes de autoeficácia e mobilizá-las com o objetivo de aumentar a autoeficácia do indivíduo face à sua capacidade para lidar de forma bemsucedida com as transições profissionais (Vieira \& Theotonio, 2018).

Concluindo, na sua globalidade, os resultados desta investigação corroboram estudos anteriores ao nível do impacto da adaptabilidade e decisão de carreira sobre a empregabilidade. A consonância com estudos anteriores vem reforçar a importância que a adaptabilidade e decisão de carreira possuem junto de adultos em transição de carreira. Contudo, nem todas as dimensões da adaptabilidade de carreira foram preditoras da empregabilidade, sendo o controlo e a confiança as dimensões que mais se destacaram quanto ao seu poder preditor da empregabilidade. De destacar que tanto o controlo como a confiança se relacionam, em termos conceptuais, com os conceitos interligados de agência e de autoeficácia cujo papel relevante no sucesso da transição para o trabalho tem vindo a ser apontado (Vieira \& Theotonio, 2018). Do ponto de vista empírico, o estudo de Gamboa e colaboradores (2014) evidenciou uma correlação de .54 entre adaptabilidade de carreira e autoeficácia na transição para o trabalho, sendo as dimensões controlo e confiança aquelas que mais contribuíram na força dessa relação (.48 e .51 , respetivamente).

Embora o presente estudo traga algumas contribuições para a teoria e para a intervenção na área das transições profissionais, também apresenta limitações. A dimensão da amostra, a sua especificidade em termos regionais e culturais, bem como o facto de todos os participantes terem procurado os serviços de consultoria de carreira, impossibilitam a generalização dos resultados obtidos neste estudo para outros contextos. Assim, apesar de várias evidências da literatura oferecerem suporte à generalidade dos resultados do presente estudo, e conforme procuramos evidenciar ao longo da discussão dos resultados, recomendamos que os mesmos sejam considerados com cautela. Outra limitação a apontar é o facto de este ser um estudo transversal, o que impede fazer afirmações sobre a causalidade das relações observadas. Ainda assim, os resultados deste estudo trazem evidências empíricas sobre o modo como a empregabilidade pode ser mantida e desenvolvida. Alguns resultados corroboram estudos prévios, enquanto outros, nomeadamente as relações entre o insight e a tomada de decisão, bem como o contributo do insight para a predição de todas as dimensões da empregabilidade, acrescentam novas contribuições à literatura na área da carreira. Além disso, os resultados do presente estudo apontam para implicações ao nível da intervenção na medida em que convocam os orientadores profissionais e/ou consultores de carreira a terem uma ideia mais clara sobre a forma como os seus clientes vivenciam os diferentes aspetos da empregabilidade e as suas dificuldades face às transições de carreira.

Ao concluir este estudo, cabe destacar que os modelos contemporâneos de carreira propõem que o indivíduo assuma um papel de "protagonista da sua carreira", ideia que, porventura, pode ser confundida com a transferência da responsabilidade pela carreira exclusivamente para o indivíduo. Torna-se então essencial clarificar que estamos cientes de que a empregabilidade não dependente unicamente de fatores individuais e o facto de não se conseguir um emprego não depende exclusivamente do próprio indivíduo. Consequentemente, a par da empregabilidade individual, urge realçar a empregabilidade do contexto, isto é, a capacidade do contexto económico e político para a criação de emprego (Vieira, 2012). Além disso, importa ter em conta os contextos de origem social e cultural do indivíduo os quais poderão exercer influência sobre a viabilidade das oportunidades laborais. Sugere-se, portanto, que novos estudos possam ampliar a forma como a empregabilidade é avaliada, incluindo fatores individuais, mas também aspetos associados ao contexto no qual os indivíduos estão inseridos.

Finalmente, destacamos a necessidade dos profissionais das áreas de orientação profissional e de carreira, assim como da gestão de pessoas, de desenvolverem programas e modelos de intervenção que contribuam para o planeamento e para a gestão da carreira dos indivíduos e que permitam promover maiores níveis de empregabilidade. É desejável que tais intervenções possibilitem e estimulem a construção de si mesmo e o desenvolvimento de comportamentos promotores da adaptabilidade e da empregabilidade ao longo da vida, as quais se constituem como um requisito central para o desenvolvimento de carreira do profissional do século XXI. 


\section{REFERÊNCIAS}

Ambiel, R. A. (2014). Adaptabilidade de carreira: uma abordagem histórica de conceitos, modelos e teorias. Revista Brasileira de Orientação Profissional, 15 (1), 15-24.

Audibert, A., \& Teixeira, M. A. P. (2015). Escala de adaptabilidade de carreira: evidências de validade em universitários brasileiros. Revista Brasileira de Orientação Profissional, 16(1), 83-93.

Bardagi, M. P., \& Hutz, C. S. (2010). Satisfação de vida, comprometimento com a carreira e exploração vocacional em estudantes universitários. Arquivos Brasileiros de Psicologia, 62(1), 159-170.

Baruch, Y., Szücs, N., \& Gunz, H. (2015). Career studies in search of theory: The rise and rise of concepts. Career Development International, 20(1), 3-20. http://doi.org/10.1108/CDI-11-2013-0137

Berntson, E., Sverke, M., \& Marklund, S. (2006). Predicting perceived employability: Human capital or labour market opportunities?. Economic and Industrial Democracy, 27, 223-244. http://doi.org/10.1177/0143831X06063098

Borges, L. L. F., De Andrade, A. L., Oliveira, M. Z. de, \& Guerra, V. M. (2015). Expanding and Adapting the Protean Career Management Scale for University Students (PCMS-U). The Spanish Journal of Psychology, 18, E103. http://doi.org/10.1017/sjp.2015.83

Bridgstock, R. (2009). The graduate attributes we've overlooked: Enhancing graduate employability through career management skills. Higher Education Research and Development, 28(1), 31-44. http://doi.org/ 10.1080/07294360802444347

Buscacio, R. C. Z., \& Soares, A. B. (2017). Expectativas sobre o desenvolvimento da carreira em estudantes universitários. Revista Brasileira de Orientação Profissional, 18(1), 69-79. http://doi.org/10.26707/1984-7270/2017v18n1p69

Campos, K. C. L., \& Freitas, F. A. (2008). Empregabilidade: construção de uma escala. Psico USF, 13(2), 189-201. http://doi.org/10.1590/S1413-82712008000200006

Cardoso, P., Duarte, M. E., \& Sousa, A.. (2016). Desenvolvimento vocacional e aconselhamento de carreira: contributos para a justiça social. Revista Brasileira de Orientação Profissional, 17(2), 257-266.

Clarke, M. (2007). Where to from here? Evaluating employability during career transition. Journal of Management \& Organization, 13, 196-211 http://doi.org/10.1017/S1833367200003692

Creed, P. A., Fallon, T., \& Hood, M. (2009).The relationship between career adaptability, person and situation variables, and career concerns in young adults.Journal of Vocational Behavior, 74, 219229. http://dx.doi.org/10.1016/j.jvb.2008.12.004.

da Costa DaSilveira, A. C., DeCastro, T. G., \& Gomes, W. B. (2012). Escala de autorreflexão e insight: Nova medida de autoconsciência adaptada e validada para adultos brasileiros. Psico, 43(2), 155-162.

Duffy, R. D., Blustein, D. L., Diemer, M. A., \& Autin, K. L. (2016). The psychology of working theory. Journal of Counseling Psychology, 63(2), 127-148. http://doi.org/10.1037/cou0000140

Fraga, S. I D. (2012). Adaptabilidade e empregabilidade numa perspectiva construtivista: Estudo com desempregados em contexto de formação [Tese de Doutoramento não publicada]. Faculdade de Psicologia, Universidade de Lisboa.

Fugate, M., Kinicki, A. J., \& Ashforth, B. E. (2004). Employability: A psycho-social construct, its dimensions, and applications. Journal of Vocational Behavior, 65, 14-38. http://doi.org/10.1016/j.jvb.2003.10.005

Gamboa, V., Paixão, O., \& Palma, A. I. (2014). Adaptabilidade de carreira e autoeficácia na transição para o trabalho: 0 papel da empregabilidade percebida-estudo com estudantes do ensino superior. Revista Portuguesa de Pedagogia, 2 (1), 133-156. http://doi.org/10.14195/1647-8614_48-2_7

Ginevra, M. C., Pallini, S., Vecchio, G. M., Nota, L., \& Soresi, S. (2016). Future orientation and attitudes mediate career adaptability and decidedness. Journal of Vocational Behavior, 95, 102-110. http://doi.org/10.1016/j.jvb.2016.08.003

Grant, A.M., Franklin, J. \& Langford, P. (2002). The self-reflection and insight scale: A new measure of private self-consciousness. Social Behavior and Personality, 30, 821-836. http://doi.org/10.2224/sbp.2002.30.8.821

Guilbert, L., Bernaud, J., Gouvernet, B. \& Rossier, J. (2016). Employability: review and research prospects. International Journal of Educational and Vocational Guidance 16, 69-89. http://doi.org/10.1007/s10775-015-9288-4

Hirschi, A., Herrmann, A., \& Keller, A. C. (2015). Career adaptivity, adaptability, and adapting: A conceptual and empirical investigation. Journal of Vocational Behavior, 87, 1-10. http://doi.org/10.1016/j.jvb.2014.11.008

Kanfer, R., Wanberg, C. R. \&Kantrowitz, T. M. (2001). Job search and employment: A personalymotivacional analysis and meta-analytic review. Journal of Applied Psychology, 86(5), 837-855. 
Leal, C. K. N., DeSouza, N. D. P. \& DeSouza, M. L. (2018). Autorreflexão e insight como dimensões da autoconsciência privada: uma revisão da literatura. Psico, 49(3), 231-241. http://doi.org/10.15448/1980-8623.2018.3.26732

Nota, L., Ginevra, M. C., \& Soresi, S. (2012). The Career and Work Adaptability Questionnaire (CWAQ): A first contribution to its validation. Journal of Adolescence, 35, 1557-1569. http://doi.org/10.1016/j.adolescence.2012.06.004

Oliveira, M. Z., \& Gomes, W. B. (2014). Estilos reflexivos e atitudes de carreira proteana e sem fronteiras nas organizações contemporâneas brasileiras. Revista Psicologia Organizações e Trabalho, 14(1), $105-118$.

Parker, D. (2008). The self in moral space. Life narrative and the good. Cornell University Press.

Pool, L. D., \& P. Sewell (2007). The key to employability: developing a practical model of graduate $\begin{array}{llll}\text { employability. } \quad \text { Education } & \text { 227-289. }\end{array}$ http://doi.org/10.1108/00400910710754435

Rizzatti, D.B., Sacramento, A.M., Valmorbida, V.S., Mayer, V.P. \& Oliveira, M.Z. (2018). Transição de carreira em adultos brasileiros: Um levantamento da literatura científica. Revista Interinstitucional de Psicologia, 11(1), 153-173. http://doi.org/10.36298/gerais2019110112

Rossier, J. (2015). Adaptability and life designing. In L. Nota, \& J. Rossier (Eds.), Life design handbook (pp. 153-167). Hogrefe.

Rossier J., Ginevra M.C., Bollmann G., \& Nota, L. (2017) The importance of career adaptability, career resilience, and employability in designing a successful life. In Maree K. (ed.) Psychology of Career Adaptability, Employability and Resilience (pp. 65-82). Springer.

Rothwell, A., Herbert, I., \& Rothwell, F. (2008). Self-perceived employability: Construction and initial validation of a scale for university students. Journal of Vocational Behavior, 73(1), 1-12. http://doi.org/10.1016/j.jvb.2007.12.001

Savickas, M.L. (2013). Career construction theory and practice. In R. W. Lent \& S. D. Brown (Eds.), Career development and counseling: putting theory and research to work (2nd ed., pp. 147-183). John Wiley \& Sons.

Savickas, M. L., \& Porfeli, E. J. (2012). Career Adapt-Abilities Scale: Construction, reliability, and measurement equivalence across 13 countries. Journal of Vocational Behavior, 80(3), 661-673. http://doi.org/10.1016/j.jvb.2012.01.011

Sok, J., Blomme, R., \& Tromp, D. (2013). The use of the psychological contract to explain self-perceived employability. International Journal of Hospitality Management, 34, 274-284. http://doi.org/10.1016/j.ijhm.2013.03.008

Stein, D. \& Grant, A. M. (2014). Disentangling the relationships among self-reflection, insight, and subjective well-being: The role of dysfunctional attitudes and core Self-Evaluations. The Journal of Psychology, 148 (5), 505-522. http://doi.org/10.1080/00223980.2013.810128

Teixeira, M. A. P. (2002). A experiência de transição entre a universidade e o mercado de trabalho na adultez jovem [Tese de Doutoramento não publicada]. Universidade Federal do Rio Grande do Sul.

Teixeira, M.A.P, \& Gomes, W.B. (2005). Decisão de carreira entre estudantes em fim de curso universitário. Psicologia: Teoria e Pesquisa, 21(3), 327-334. http://doi.org/10.1590/S010237722005000300009

Teixeira, M. A. P., Oliveira, M. C., Melo-Silva, L. L., \& Taveira, M. C. (2019). Escalas de desenvolvimento de carreira de universitários: Construção, características psicométricas e modelo das respostas adaptativas. Revista Psicologia: Organizações e Trabalho, 19(3), 703-712. http://doi.org/10.17652/rpot/2019.3.16557

Vieira, D.A. (2012). Transição do ensino superior para o trabalho: O poder da autoeficácia e dos objetivos profissionais. Politema/Vida Económica.

Vieira, D. A., \& Theotonio, M. (2018). Autoeficácia e sucesso na transição para o trabalho: um estudo longitudinal. Revista Brasileira de Orientação Profissional, 19(1), 31-39. http://doi.org/1026707/1984-7270/2019v19n1p31

Zanon, C., Teixeira, M. A. P. (2006). Adaptação do Questionário de Ruminação e Reflexão (QRR) para estudantes universitários brasileiros. Interação em Psicologia, 10(1), 75-82.

Historial do artigo

Recebido $\quad 11 / 2019$

Aceite $\quad 07 / 2020$

Publicado $12 / 2020$ 
Preditores da empregabilidade 